\title{
3D MODELING OF ULTRASONIC WAVE INTERACTION WITH DISBONDS AND WEAK BONDS
}

\author{
C. Leckey ${ }^{1}$, M. Hinders ${ }^{2}$ \\ ${ }^{1}$ NASA Langley Research Center, Hampton, VA 23681 \\ ${ }^{2}$ Department of Applied Science, College of William and Mary, Williamsburg, VA 23187
}

\begin{abstract}
Ultrasonic techniques, such as the use of guided waves, can be ideal for finding damage in the plate and pipe-like structures used in aerospace applications. However, the interaction of waves with real flaw types and geometries can lead to experimental signals that are difficult to interpret. 3dimensional (3D) elastic wave simulations can be a powerful tool in understanding the complicated wave scattering involved in flaw detection and for optimizing experimental techniques. We have developed and implemented parallel 3D elastodynamic finite integration technique (3D EFIT) code to investigate Lamb wave scattering from realistic flaws. This paper discusses simulation results for an aluminum-aluminum diffusion disbond and an aluminum-epoxy disbond and compares results from the disbond case to the common artificial flaw type of a flat-bottom hole. The paper also discusses the potential for extending the 3D EFIT equations to incorporate physics-based weak bond models for simulating wave scattering from weak adhesive bonds.
\end{abstract}

Keywords: Ultrasonic, simulation, bond

PACS: 43.20.El,43.35.Zc,43.40.Le

\section{INTRODUCTION}

Modeling of ultrasonic wave behavior in materials containing flaws has long appeared in the literature in the field of nondestructive evaluation. The modeling approaches range from analytical techniques to numerical and hybrid modeling methods [1]. There are several common numerical methods including boundary element method (BEM), finite element method (FEM), and finite difference techniques (FD). Our current work focuses on elastodynamic finite integration technique (EFIT), which is similar to elastodynamic staggered-grid finite difference time domain (FDTD) techniques. EFIT was first reported in the literature in the early 1990s by Fellinger and Langenberg [2]. Since that time, EFIT has been used to simulate various NDE situations, such as Lamb wave propagation in pipes, wave interaction with cracks, and waves in anisotropic material [3-5]. A review of the history of finite integration technique can be found in [6].

We have developed a parallel 3D EFIT code that runs on a computing cluster. The parallel code uses a 1D virtual topology for partitioning the grid onto different CPUs, thus requiring corresponding stress and velocity variables to be passed between computer processors using Message Passing Interface (MPI). In our previous work we compared 3D EFIT results to both analytical results and experimental data [7]. In this paper we will discuss our preliminary work with using EFIT to model wave interaction with disbonds and weak bonds. The EFIT equations for an inhomogeneous isotropic material can be found in 
several references, such as [2], and will not be repeated here. The technique involves integrating the elastodynamic equations of motion over a control volume, which is assumed to be a cube for the Cartesian case that we have implemented. The method leads to six equations for stress, $\mathrm{T}_{\mathrm{ij}}$, and three equations for velocity, $\mathrm{v}_{\mathrm{i}}$, that are discretized in time and space.

\section{WAVE INTERACTION WITH DISBONDS}

Diffusion bonding is a technique that can be used to join similar or dissimilar materials by applying pressure for a specified amount of time at elevated temperatures $[8,9]$. The quality of the resulting bond depends on these factors, as well as the surface condition of the two materials. Non-ideal conditions can create disbonds (voids) between the materials [10]. Even well-bonded materials can develop voids if the specimen is subject to an impact or other outside stresses [9].

We have simulated scattering from a disbond in diffusion bonded aluminum 2024 plates with a combined thickness of $3.15 \mathrm{~mm}$. The disbond is introduced into the simulation by setting stress-free boundary conditions in the desired region. We chose to create a disbond void that is a rounded-rectangle in shape (see figure 1), to allow for comparisons to our previous work in simulating wave interaction with rounded-rectangle flat-bottom holes in aluminum [7]. The thickness of the simulated void is 195 microns, and represents a case where the void thickness has been enlarged due to external applied stresses. The incident wave was a 5-cycle $2.15 \mathrm{MHz}$ pulse introduced using a round $7 \mathrm{~mm}$ diameter transmitter.

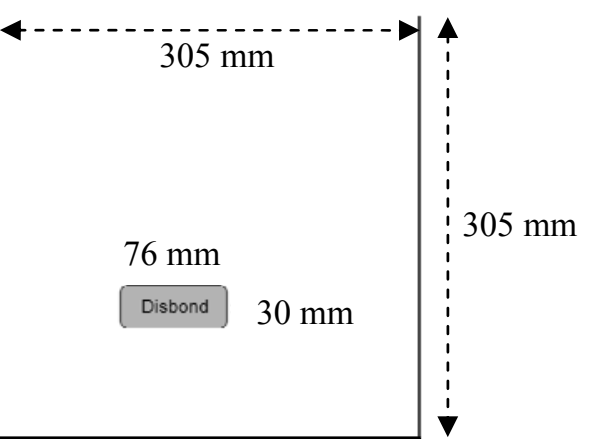

FIGURE 1. Diagram showing the rounded rectangle disbond shape and location on the aluminum plate.

The EFIT output images shown in this work represent the out-of-plane motion, $\mathrm{v}_{3}$, again for comparison to our previous work in which EFIT flat-bottom hole simulations were compared to experimental data from a longitudinal transducer. The disbond case differs from the flat-bottom hole case by the fact that material now exists above the void region, allowing for wave propagation in that region. We performed several simulations, changing the disbond depth and transmitter location. In this paper we give one example case of our results. The results are shown as EFIT images and spatial domain short time Fourier transform (spatial spectrogram) images of the EFIT data. The spatial spectrogram technique leads to plots of wavenumber versus position, and allow us to track mode conversion in the simulated data as the waves propagate.

The results discussed here are for a disbond located at a depth of approximately 1.29 $\mathrm{mm}$. The regions above and below the disbond have frequency-thickness products of 3.55 MHz-mm and 2.77 MHz-mm. EFIT images and the corresponding spatial spectrogram 
plots for a single transmitter position are shown in Figures 2-4. The transducer for those figures is located at $0.095 \mathrm{~m}$ on the $\mathrm{y}$-axis and would be located at $0.035 \mathrm{~m}$ on the $\mathrm{x}$-axis of that Figure. The spatial spectrogram plots in Figure 3 a) and b), taken directly beneath the disbond, show the emergence of two of the three expected Lamb modes for the lower material thickness. The A0 mode does not emerge, possibly due to disruption caused by scattering effects. Above the void, the spectrograms in Figure 4 a) and b) show the presence of all five expected Lamb modes, however, the S0 mode disappears as it travels over the disbond region. After the flaw region we do not see the re-emergence of all eight expected modes, see Figures 3-4 c) and d). The S0 and A0 modes do not reappear after encountering the disbond. We will not show the flat-bottom hole results here, but for the sake of comparison, we mention that in the case of a flat-bottom hole of depth $1.29 \mathrm{~mm}$ we observed the re-emergence of all expected Lamb modes [7]. Based on observations of EFIT simulation output for progressing points in time, it seems that the disappearance of these two modes may be caused by wave interference during the re-combining of upper and lower plates.
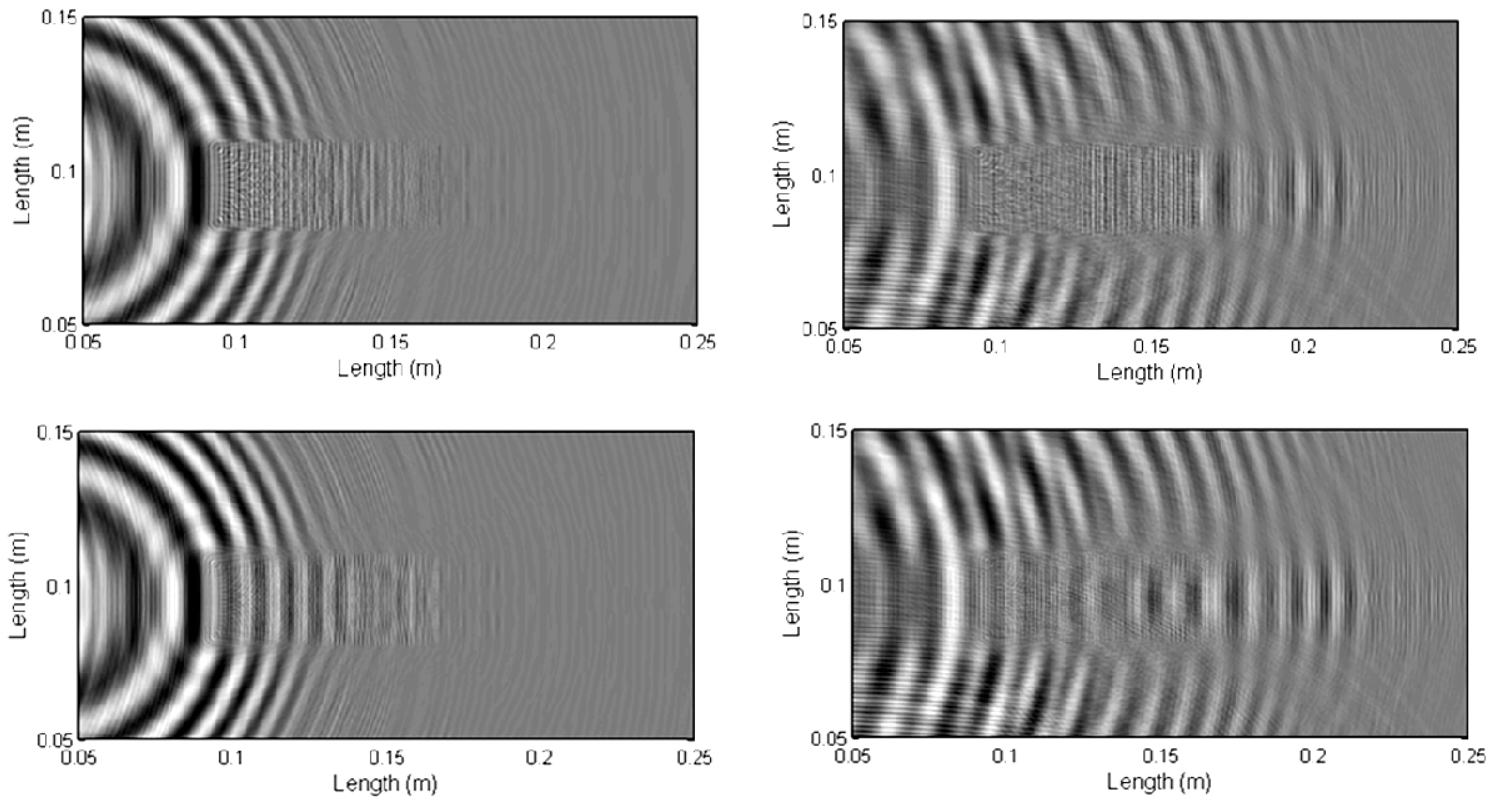

FIGURE 2. EFIT results for bonded Al plates with a disbond located at a depth of $1.29 \mathrm{~mm}$ for the transmitter position described above. The images show wave propagation in a portion of the plate at two points in time, top images: 2D slices taken through the 3D simulation space at a position directly beneath the disbond at $\mathrm{t}=45 \mu \mathrm{s}$ (left) and $\mathrm{t}=81 \mu \mathrm{s}$ (right), bottom: $2 \mathrm{D}$ slices taken direction above the disbond at $\mathrm{t}=45 \mu \mathrm{s}$ (left) and $\mathrm{t}=81 \mu \mathrm{s}$ (right).

\section{WAVE INTERACTION WITH WEAK BONDS}

Typical types of adhesive bonds found in aerospace applications are aluminumepoxy bonds and adhesively bonded composites. One key benefit of adhesive bonding over traditional methods, such as the use of rivets, is that adhesive bonds lead to more uniform stress distributions [11]. Yet, the use of adhesive bonds also makes a component susceptible to damage/flaws that are difficult to detect. The three defects commonly encountered with adhesive bonds are disbonds between the adherend and adhesive (voids), weak adhesive layers (low cohesive strength), and a weak bond between the adhesive and adherend [12]. 
The last of these is reportedly particularly difficult to detect since the interface layer between the adherend and adhesive is only on the order of a few microns thick [13].

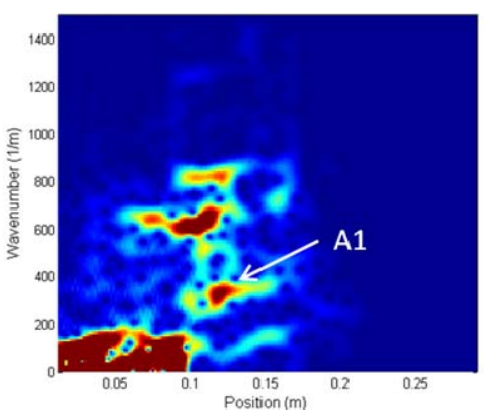

a)

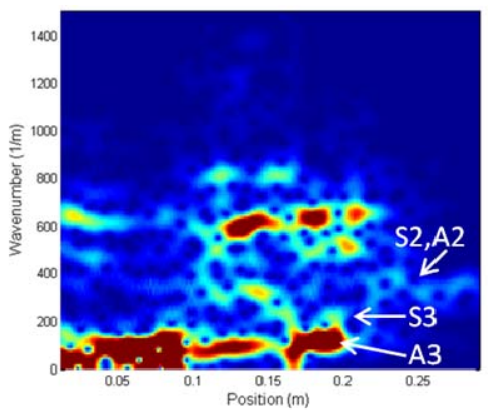

c)

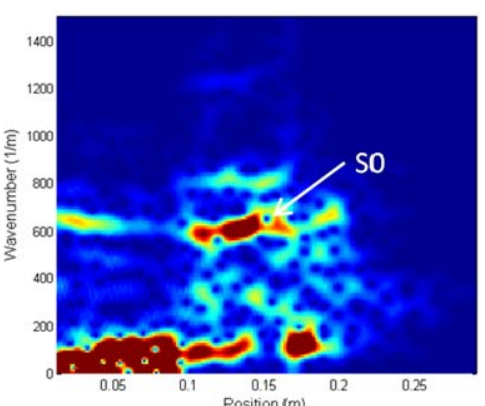

b)

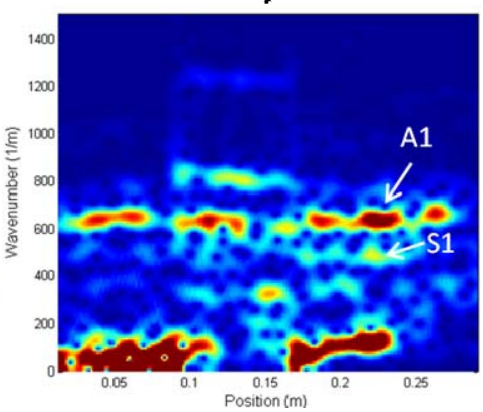

d)

FIGURE 3. Spatial spectrogram plots (showing wavenumber versus position) created from an A-line directly beneath the void, through the center of the images in Figure 2, for times a) $t=45 \mu \mathrm{s}, b$ ) $t=63 \mu \mathrm{s}, \mathrm{c}) \mathrm{t}=72 \mu \mathrm{s}$, and d) $\mathrm{t}=90 \mu \mathrm{s}$. The region parallel to the disbond extends from approximately $0.09 \mathrm{~m}$ to $0.16 \mathrm{~m}$. Observed Lamb wave modes are labeled, and mode conversion parallel to the disbond can be seen.

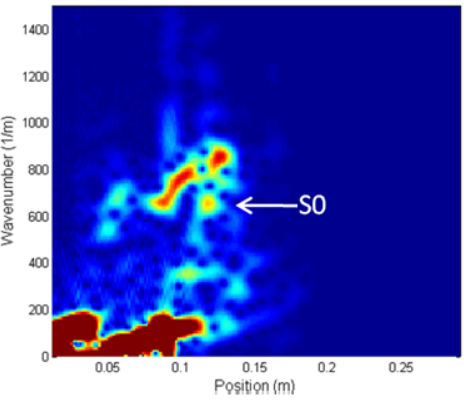

a)

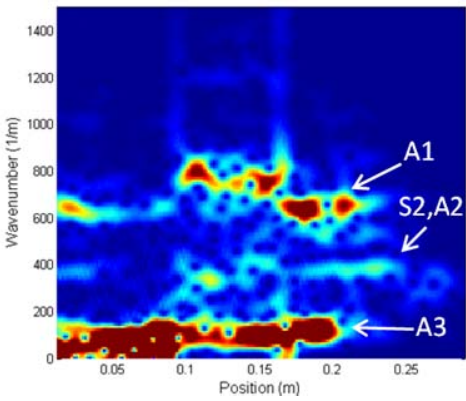

c)

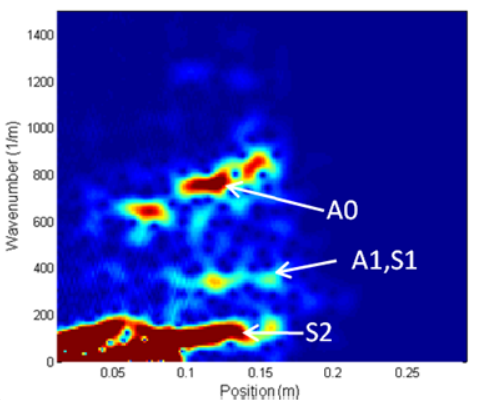

b)

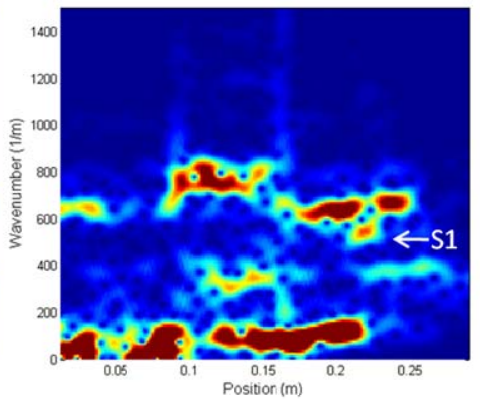

d)

FIGURE 4. Spatial spectrogram plots (showing wavenumber versus position) created from an A-line directly beneath the void, through the center of the images in Figure 2, for times a) $t=36 \mu \mathrm{s}, b) t=45 \mu \mathrm{s}, \mathrm{c}) \mathrm{t}=63 \mu \mathrm{s}$, and d) $\mathrm{t}=72 \mu \mathrm{s}$. The region parallel to the disbond extends from approximately $0.09 \mathrm{~m}$ to $0.16 \mathrm{~m}$. Observed Lamb wave modes are labeled, and mode conversion parallel to the disbond can be seen. 
Various ultrasonic techniques for detecting a weak interface bond are reported in the literature, such as: the use of Lamb waves, guided modes in the adhesive layer, measurement of reflection coefficients from adhesive/adherend interface, and angle beam ultrasonic spectroscopy [13-15]. Realistic simulations of ultrasonic waves interacting with weak bonds would provide an understanding of this complex behavior and could be used to develop and optimize nondestructive detection techniques. Previous work in the field of modeling weak bonds has involved approaches such as transitioning from welded to slip boundary conditions at the interface using spring boundaries [16-18]. Contact-friction interfaces and periodic air or water filled voids are also approaches that have been used to model weak bonds [11]. Incorporating a simple change of boundary conditions into the EFIT code is a straight-forward task, making the technique ideal for incorporating weak bond models.

Figure 5 shows an example comparison of EFIT results for two cases of aluminumepoxy-aluminum bonding: a) full contact, and b) slip boundaries along the top interface. The full sample thickness is $3.15 \mathrm{~mm}$, matching with the disbond case we discussed above. The images are shown purely as an example of the subtle differences resulting in the EFIT simulation for the two cases. Slightly larger disruption of waves occurs in the case with slip boundaries along the top interface surface.

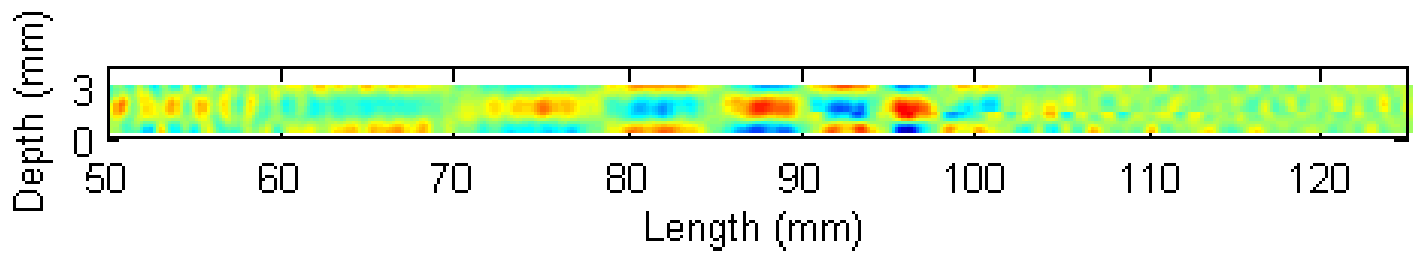

a)

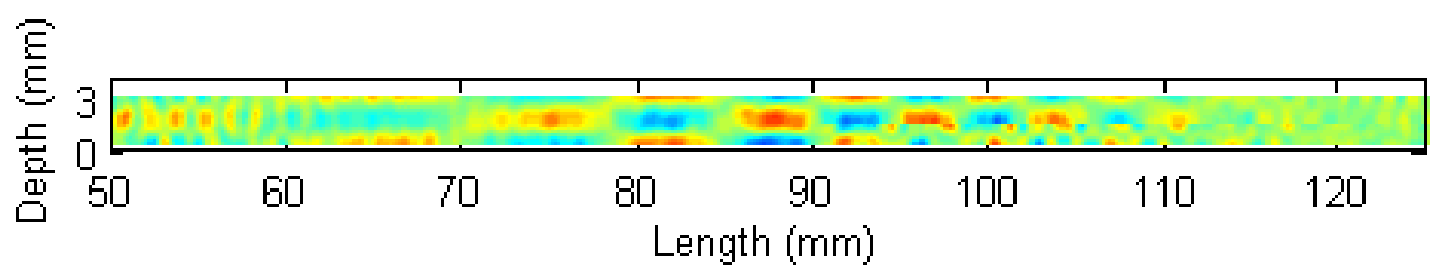

b)

FIGURE 5. EFIT thickness plots of $v_{3}$ at a single point in time, showing Lamb wave behavior in aluminum plates with an epoxy layer that begins around $90 \mathrm{~mm}$ on the $\mathrm{x}$-axis and extends to the end of the image. The transducer would be located at $35 \mathrm{~mm}$ on these plots, and the waves in the snapshots shown above are moving from left to right. a) Full contact between the aluminum and epoxy layer, b) A large portion of the top aluminum-epoxy interface has slip boundary conditions applied.

\section{ANGLE BEAM TECHNIQUES}

Since angle beam ultrasonic spectroscopy techniques have proven successful at detecting weak adhesive bond interfaces, in future work we plan to simulate wave interaction due to angled incident beams. An angled incident beam can be introduced into EFIT by introducing a delay in the input signal as we move across EFIT grid points corresponding to the transducer. The technique is analogous to phased array beam forming. 
Further details on angled beams with EFIT can be found in work by Halkjaer [5]. The time delay, $\mathrm{t}_{\text {delay, }}$ necessary to direct the beam at an angle, $\theta$, is given below:

$$
t_{\text {delay }}=\frac{\left(n-n_{\min }\right) \Delta x}{c_{r e f r} \Delta t} \sin \theta_{\text {refr }}
$$

where $n$ represents the current grid index, $n_{\min }$ is the minimum grid index associated with the transducer location, $\Delta x$ and $\Delta t$ are the spatial and temporal step sizes, $c_{r e f r}$ is the speed of sound in the medium (refracted speed of sound), and $\theta_{\text {refr }}$ is the desired refracted (beam direction) angle [5]. The images below show an example of an angled beam incorporated into an EFIT simulation with stress-free boundaries along the simulation edges.

Tme 1

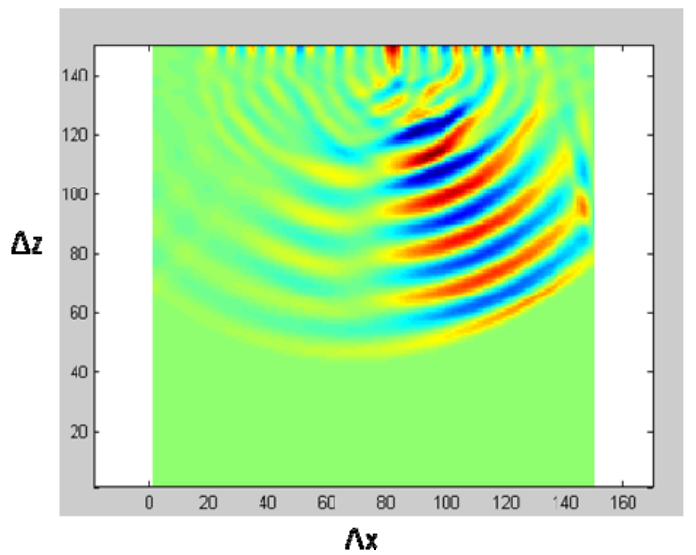

Time 2

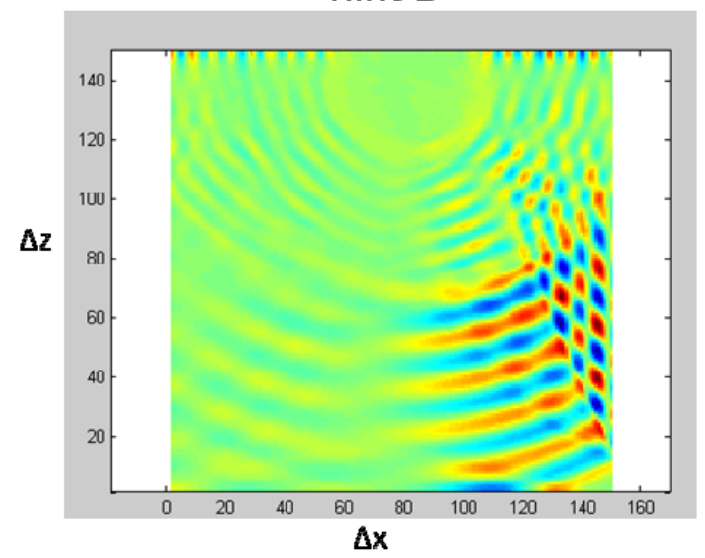

FIGURE 6. $2 \mathrm{D}$ slices taken through the $3 \mathrm{D}$ EFIT simulation space showing waves propagating in a cube at two points in time. The angled incident beam was introduced into the simulation via equation (1).

\section{CONCLUSION}

The work described above shows that EFIT is a useful tool for investigating complicated ultrasonic wave interaction with 3D flaws. In our future work we hope to further incorporate weak bond models into the EFIT simulations. In particular, we plan to focus on models with a direct link to the physics involved in describing disbonds on a molecular level. Additionally, we plan to use the time delay technique to explore 3D angled beam ultrasonic wave interactions with weak adhesive bond interfaces.

\section{ACKNOWLEDGEMENTS} techniques.

The authors thank Dr. Matt Rogge for helpful discussions on spatial spectrogram

\section{REFERENCES}

1. J.L. Rose. Ultrasonic Waves in Solid Media. Cambridge University Press, 1999.

2. F. Fellinger and K.J. Langenberg. Numerical techniques for elastic wave propagation and scattering. Proc IUTAM Symp, pages 81-86, July-Aug 1990. 
3. Kevin Rudd, Kevin Leonard, Jill Bingham, and Mark Hinders. Simulation of guided waves in complex piping geometries using the elastodynamic finite integration technique. J Acoust Soc Am, 121(3):1449-1458, 2007.

4. Frank Schubert, Bernd Koehler, and Pavel Zinin. Numerical time-domain simulation of wave propagation and scattering in acoustic microscopy for subsurface defect characterizaiton. Proc SPIE, 5766(106):106-117, 2005.

5. S. Halkjaer. Elastic wave propagation in anisotropic, inhomogeneous materials: application to ultrasonic NDT. PhD Thesis, Technical University of Denmark, 1999.

6. R. Marklein. The finite integration technique as a general tool to compute acoustic, electromagnetic, elastodynamic, and coupled wave fields. In W.R. Stone, editor, Review of Radio Science: 1999-2002 URSI, pp 201-244. IEEE Press and John Wiley and Sons, New York, 2002.

7. C. Leckey. Investigation of ultrasonic wave scattering effects using computational methods. Ph.D. Dissertation, College of William and Mary, 2011.

8. A. Hill and E.R. Wallach. Modelling solid-state diffusion bonding. Acta Metall, 37(9):2425-2437, 1989.

9. Horng-YuWu, Shyong Lee, and Jian-YihWang. Solid-state bonding of iron-based alloys, steel-brass and aluminum alloys. Materials Processing Technology, 75:173-179, 1998.

10. M. Joseph Fernandus, T. Senthilkumar, and V. Balasubramanian. Developing temperature-time and pressure-time diagrams for diffusion bonding AZ80 magnesium and AA6061 aluminum alloys. Mater Des, 32:1651-1656, 2011.

11. S. Yang, L. Gu, and R. Gibson. Nondestructive detection of weak joints in adhesively bonded composite structures. Composite Structures, 51:63-71, 2001.

12. S. Yang, R.F. Gibson, L. Gu, and W.H. Chen. Modal parameter evaluation of degraded adhesively bonded composite beams. Composite Structures, 43:79-91, 1998.

13. Peter Cawley. Ultrasonic measurements for the quantitative NDE of adhesive joints potential and challenges. Ultrasonics Symp, pages 767-772, 1992.

14. F.J. Margetan, R.B. Thompson, J.H. Rose, and T.A. Gray. The interaction of ultrasound with imperfect interfaces: Experimental studies of model structures. J Nondestruct Eval, 11:102-126, 1992.

15. L. Adler, S. Rokhlin, and A. Baltazar. Determination of material properties of thin layers using angle beam ultrasonic spectroscopy. IEEE Ultrason Symp, pages 701-704, 2001.

16. A.I. Lavrentyev and S.I. Rokhlin. Models for ultrasonic characterization of environmental degredation of interfaces in adhesive joints. J Appl Phys, 76(8):46434650, 1994.

17. W.Q. Chen, Y.F. Wang, J.B. Cai, and G.R. Ye. Three-dimensional analysis of cross-ply laminated cylindrical panels with weak interfaces. Solids and Structures, 41:2429-2446, 2004.

18. Q.B. Han, M.L. Qian, and H. Wang. Investigation of solid/solid interface waves with laser ultrasonics. Ultrasonics, 44:1323-1327, 2006. 